See discussions, stats, and author profiles for this publication at: https://www.researchgate.net/publication/256460775

\title{
Time to Change: Deciding When to Switch Action Plans during a Social Interaction
}

Chapter · August 2013

DOI: 10.1007/978-3-642-39802-5

\section{CITATIONS}

4 authors:

Eris Chinellato

Middlesex University, UK

83 PUBLICATIONS 1,099 CITATIONS

SEE PROFILE

Luisa Sartori

University of Padova

71 PUBLICATIONS 1,815 CITATIONS

SEE PROFILE
READS

124

Dimitri Ognibene

Università degli Studi di Milano-Bicocca

65 PUBLICATIONS 713 CITATIONS

SEE PROFILE

Yiannis Demiris

Imperial College London

247 PUBLICATIONS 5,317 CITATIONS

SEE PROFILE

Some of the authors of this publication are also working on these related projects:

Project The role of attention in the context of complementary interactions View project

Project Robot-Assisted Dressing View project 


\title{
Time to Change: Deciding When to Switch Action Plans during a Social Interaction
}

\author{
Eris Chinellato ${ }^{1}$, Dimitri Ognibene ${ }^{1}$, Luisa Sartori $^{2}$, and Yiannis Demiris ${ }^{1}$ \\ 1 Department of Electrical and Electronic Engineering, Imperial College London, UK \\ 2 Department of General Psychology, University of Padova, Italy \\ e.chinellato@imperial.ac.uk
}

\begin{abstract}
Building on the extensive cognitive science literature on the subject, this paper introduces a model of the brain mechanisms underlying social interactions in humans and other primates. The fundamental components of the model are the "Action Observation" and "Action Planning" Systems, dedicated respectively to interpreting/recognizing the partner's movements and to plan actions suited to achieve certain goals. We have implemented a version of the model including reaching and grasping actions, and tuned on real experimental data coming from human psychophysical studies. The system is able to automatically detect the switching point in which the Action Planning System takes control over the Action Observation System, overriding the automatic imitation behaviour with a complementary social response. With such computational implementation we aim at validating the model and also at endowing an artificial agent with the ability of performing meaningful complementary responses to observed actions in social scenarios.
\end{abstract}

Keywords: Social interaction, motor simulation, action observation, action planning, motor primitives.

\section{Introduction}

While observing a partner executing an action, it appears that the observer's motor system is pre-activating for the execution of the same action 112112. In most cases, the motor signal is never released, and such activation remains mostly unconscious (but measurable by neurophysiological experimental techniques 181614). The consequences of this phenomenon for the development of the motor system and the acquisition of social skills are nevertheless fundamental. In fact, this well recognised cognitive mechanism constitutes a typical "mirror" effect, and elicits phenomena such as understanding and interpretation of a partner's actions 2011. Such resonance represents also a natural substrate for imitation behaviors [13]. When required though, the motor system will stop resonating, for preparing a complementary action response [1819.

Thus, two strictly interrelated processes, which have been called Action Observation System (AOS), and Action Planning System (APS), are contextually active during social interactions [3]. The APS is the neural system which, 
using proprioception and sensory input regarding the surrounding environment, and according to the subject personal objectives and motivations, plans and monitors the execution of all sort of actions. The AOS is instead in charge of following the actions of a partner, mainly by matching them to a subject own motor repertoire.

In this work we investigate the nature of the link between AOS and APS, exploring their behavior in different environmental and social conditions. We model both Action Observation and Action Planning systems with competitive structures, in which candidate actions composed by pairs of inverse and forward models are dynamically evaluated and compared, in the first case referred to observed actions, in the second to planned ones. We have been testing interaction mechanisms between the systems which can explain the effects described in the literature, while constituting the base for the generation of skilled complementary responses in human-robot social setups. With our model, we are able to reproduce the mirroring to complementary switching effect observed in human studies. Such skill can been applied to actual interaction data in which human movements are tracked and interpreted in real-time by a robotic system, in order to interpret the subject actions and prepare an appropriate response.

\section{Modeling AOS and APS}

Our model is based on the competition among candidates composed by pairs of inverse and forward models. Similar frameworks, based on the concepts of competition, simulation and hierarchy, have been successfully applied to various behavioural tasks in the past [1022, but the introduction of a dual competitive system is completely novel, and allows to explain a number of neuroscience findings difficult to justify otherwise.

Three inverse/forward model pairs in a competitive framework can be observed in Fig,1] An inverse model computes the motor plan required to achieve a target state considering the current state. A forward model estimates the next state, given the current state and the motor plan. Coupling inverse and forward models allows the motor system to perform a feed-forward control in order to anticipate the evolution of an action and its effects on the environment. In a competitive system, such as that of Fig, 1, the accuracies of various inverse/forward model pairs in predicting the next state can be compared in order to assess what pairs constitute the most suitable representations of the ongoing phenomenon. More precisely, in the case of APS, the most accurate model pair is the one best suited to achieve the goal state from the initial state. The motor plan devised by the most accurate inverse model is forwarded to the motor cortex. If a motor signal is finally released, such plan is thus employed by the subject in order to actually achieve the goal state. In the AOS case, the most accurate model pair is the one which is best at capturing the action performed by a partner. It is important to clarify that we use our own motor system, i.e., the models we have learnt by pursuing our own goals, in order to represent and interpret environment and movements of the partner 844 . We believe that, in many conditions and as a 


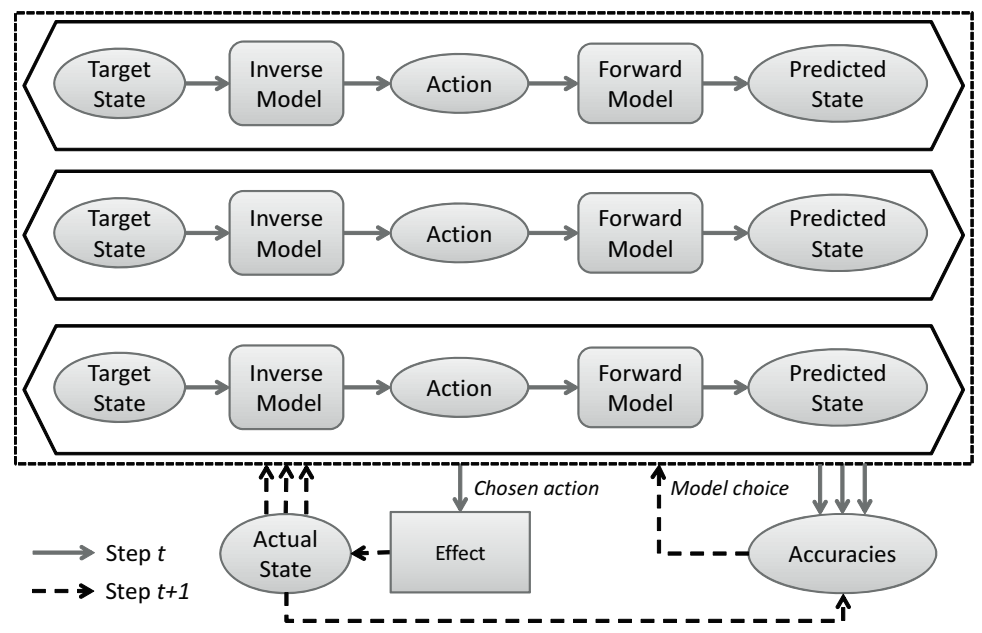

Fig. 1. Competitive framework among a number of candidate Inverse/Forward model pairs concurring for prediction and control of executed and observed actions

default behavior, the output of the most accurate inverse model is again sent to the motor cortex, and constitutes the repeatedly registered automatic imitation or motor resonance [13. In most cases, the release signal for this motor plan is never released, and the activation remains mostly unconscious.

\section{Action Primitives}

The inverse models in the above described framework represent motor primitives which are likely maintained in the premotor cortex of humans and other primates. A higher level, goal-based representation of such motor primitives is properly coded by the posterior parietal cortex [15]. This more abstract representation is instantiated with specific boundary conditions corresponding to the current state of the environment. So, e.g., the general primitive reaching is instantiated with the final objective position of the end effector, probably but not necessarily corresponding to the presence of an object. The same general primitive can also generate different inverse models in the premotor cortex, such as when multiple potential goal objects are available for a reaching action.

Primitives are organised hierarchically, and can be merged in time and space to form more complex ones. For example, a grasping action is composed by a transport and a grip components, which in turn are formed by more basic primitives (such as move arm forward, adduct a finger, and so on). The complete set of primitives of different complexity levels represent the whole motor repertoire available to a subject (see e.g. the very simplified representation in Fig 2). 


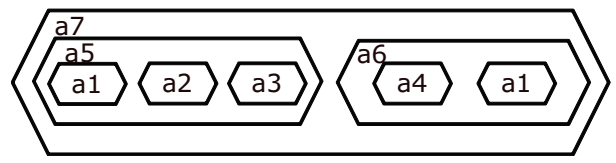

Fig. 2. Schematic representation of the structure of a possible, simple motor repertoire available to a subject

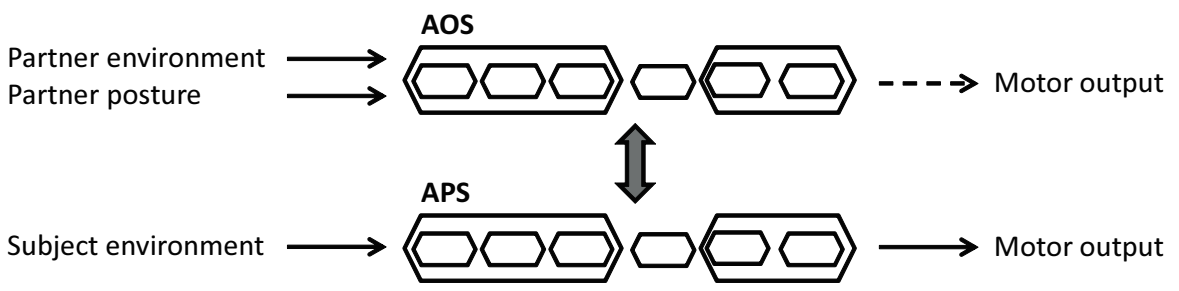

Fig. 3. General framework of the AOS/APS model

\section{AOS and APS Interacting}

AOS and APS are composed by matching primitives of the type described above. According to our model, the motor cortex can receive activation signals from either of the two systems. The influence of each system on the planned motor activity depends on both environmental and social variables, such as presence of objects in the common workspace, availability of gaze information, instruction to perform a collaborative task. All these aspects constitute triggers for switching between the two possible modalities. AOS and the typical mirror effects it elicits represent the default behaviour of the compound system, which by default resonates with the partner's actions. On the other hand, following suitable cues, APS can take charge over AOS, and the candidates which are directly related to the real spatial configuration of the observer prevail. Monitoring of the partner's movements is still be performed by AOS, in a decoupled way, and can directly affect on-line action execution if necessary.

The described experimental setup below, testbed for our model, constitutes also a typical example of the way the two systems work and interact.

\section{Experimental Setup}

As a first approximation to the objectives of our model, we have implemented a version of it which plans either Whole Hand or Precision grips on objects according to the environmental and social context and the movements of a visible partner. To test the behaviour of our model we have taken the data of a real psychophysical experiment designed to analyse what motor response subjects are preparing during different stages of a social interaction [18. 


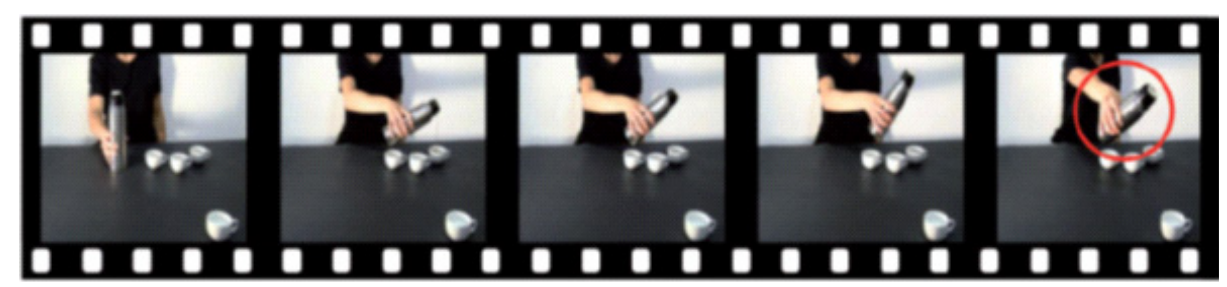

Fig. 4. Sequence from the social interaction video shown to the subjects of the experiment. The rightmost frame shows the moment in which subjects begin to interpret the movements of the demonstrator as requiring a complementary response which overcome the default resonating behaviour. Adapted from Sartori et al. 18.

The subjects of the experiment observe a video showing an actor performing a sequence of movements, representing a social interaction. Fig. 4 shows a sequence of the video of one of the conditions of the experimental protocol, in which the last frame represents a change in social requirement from the demonstrator. In the condition shown in Fig. 4 the actor/experimenter pours coffee from a thermos into three cups placed at reachable distance for her, and finally move her hand towards a fourth cup which is out of reach for her, and closer to the observer. It was shown in [18] that the motor system of the observer pre-activates for a whole hand grip, similar to that required to grasp the thermos, while the experimenter is pouring coffee into the three close cups. It thus appears that the observer motor system is resonating with the partner's at this stage. When the actor extends her hand to reach the far cup (last frame), the observer motor pre-activation changes, switching to a precision grip, suitable to grasp the fourth cup. This apparently happens so that the observer can prepare for a complementary movement - approach the cup to the partner - which represents a natural social response. It is worth noting that, in an experimental condition not shown here, pre-activation does not change if the experimenter move the hand back towards herself, without approaching the far cup. Thus, soon after a qualitative change in the nature of the social interaction, subjects switch their motor plan from mirroring to complementary.

Fig. 5 depicts the trajectory of the demonstrator hand, approximately from her own viewpoint, during the whole video. Relevant time-steps are highlighted by labeled empty circles, representing: (1) movement start; (2) thermos grasp (first frame of Fig. 4); (3) (4) (5) coffee pouring into the three close cups (second to fourth frames of Fig. 4); (6) movement end (last frame of Fig. 4). The filled round marker represents the point in time at which human experiments showed that subjects had switched from the default resonating behavior (AOS dominance) to the preparation of a complementary social response (APS dominance) [18]. The filled square marker shows the same switch point as detected by our model, as explained in Section 5 . 


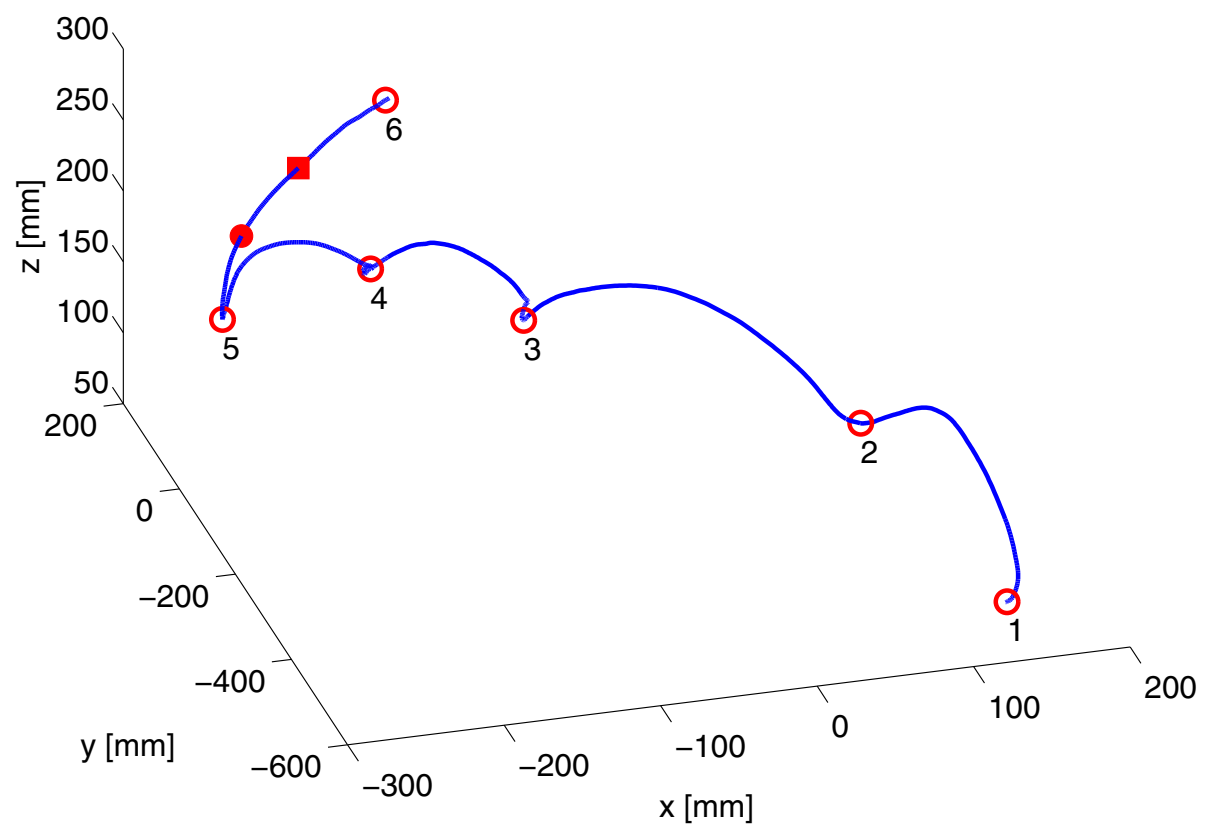

Fig. 5. Trajectory in 3D of the demonstrator hand during the whole video. The filled round marker is the point in time at which subjects were found to have switched from resonating to complementary motor response. The filled square marker shows the same switching point as detected by our model.

\section{Implementation}

The model implementation thus far consists of three main components. The first component is a representation of the motor primitives available to the subject, the second component is the competitive structure, common to both AOS and APS, and the third component is the associative memory relating action observation to action production, which takes also into account contextual information.

\subsection{Motor Primitives}

Motor primitives for reaching and grasping, respectively, are implemented with two artificial neural networks. We represent a human arm with three degrees of freedom, two for the shoulder (one for flexion/extension, the other for abduction/adduction) and the third for the elbow extension. The hand is represented with two degrees of freedom, one for the first dorsal interosseus (FDI) muscle, serving index finger flexion/extension, and thus participating in all grasping actions, and the other for the abductor digit minimi (ADM) muscle, serving little finger abduction, and hence only participating in Whole Hand grips. 
The neural networks (standard feed-forward back-propagation ANNs) are trained simulating autonomous exploration of the environment, mapping proprioceptive information on joint angles with egocentric position of the target position. Training of the arm reaching network is done by providing egocentric end effector position in input and arm joint values in output. It have thus three inputs and three outputs. The hand grasping network receives in input the size of the object and outputs the state (yes/no) of the FDI and ADM muscles required to grasp that object.

For these experiments we have omitted the processing of visual information and its mapping to joint space representation. In fact, all of the above can be done in a biological plausible way, similarly to how it is performed in the Posterior Parietal Cortex, without the need to employ Cartesian coordinates [5].

\subsection{Competition}

Competition among candidates is performed according to the principles described in Sec. 2] separately for AOS and APS, and concurrently for different muscles. This means that, in each system, competition among reaching candidates and competition among grasping candidates, although clearly related, are performed in parallel.

A fundamental point, for both its practical and theoretical implications, is the choice of the candidates that are competing at any one moment. For APS, the list of candidates is obtained from the set of objects visible to the observer. Out of reach, or even unachievable objects such as those visible on a video, are valid candidates for the grasping competition, as indicated by studies showing neural pre-activation upon simple visualization of graspable objects 9 . It has not been as consistently shown how this principle applies to reaching primitives, and we propose that the mechanism is only slightly different. In our framework, no pre-activation is possible, and thus no candidates available, for reaching actions that are impossible or unattainable in the current conditions (e.g. objects on a video), but reaching candidates are generated for those objects that are just out of reach for the subject, implying a tentative reaching or an "ask" signal. AOS candidates are generated similarly to APS's, but considering the partner's reference frame in terms of object position and graspability. There is a critical issue here though, which is that, at the motor primitives level, the observer represents the world of the partner in his own egocentric coordinates. In other words, the above means that the subject takes the perspective of the partner and represent her world as he were in her position. This perspective taking mechanism, requiring a transformation from allocentric to egocentric coordinates by the observer, seems to be performed by a network connecting parietal areas and Premotor Cortex [14. The important consequence of such transformation is that the subject can employ his own motor primitives to evaluate the movements of the partner.

To exemplify the generation of candidates and their competition, let us consider the case of the experimental setup described in Sec. 3. For what concerns grasping, all visible objects are represented in terms of the types of grasp they 
afford, and visual to motor transformation for grasping at this stage is performed as described in [76. Candidates are thus Precision or Whole Hand Grips, which will dominate according to what object is or is expected to be the action target (PG for spoon, WHG for mug). The representation is the same for subject and observer. For what concerns reaching, objects are represented both relatively to the subject point of view (in the APS) and to the partner's point of view (in the AOS). Candidates are thus the location of the potential targets in the two different egocentric spaces. Objects farther than the maximum arm extension are labeled as out of reach.

\subsection{Social Associative Memory}

The relation between AOS and APS is coordinated by a social associative memory which matches certain actions to their natural social response, irrespective of who is actually performing the action. If action B (e.g. take) usually follows action A (e.g. give), the observation of a partner executing A elicits the pre-planning of B by the observer. On the other hand, If the subject executes $\mathrm{A}$, he expects to see the partner performing $\mathrm{B}$ in response. A different response would either be classified as an anomaly to discard, or instead constitute an important new relation worth to be memorised. In any case, it is the comparison between predicted and observed stimuli, both on the personal and the social side, which drives the use and plastic modification of action components and their relations.

A social memory of this kind could be stored in the Hippocampus, but its management according to the contextual states of environment and interaction is most likely performed by the Pre-Frontal Cortex [16. Indeed, the medial Pre-Frontal Cortex (mPFC) has been observed to be more responsive during observation of social movements than individual movements 1 .

In this work, we taught our system that when a partner extends her hand towards a target that is not reachable for her (e.g. the furthest, out of reach object), she most likely expects our reaction in terms of handing her that same object. This makes the subject move its focus from the spoon (mirroring effect) to the cup closer to himself (complementary response). This switching generates an automatic change in grasp planning 97, immediately reflected by different activations of ADM and FDI muscles.

\section{Results}

The implementation of reaching primitives described above, although simple, allows us to obtain interesting results that clearly indicate the direction to follow in the next model development steps. Detection of the switching point between the dominance phases of AOS and APS by the model occurs when the actor/partner performs a reaching actions toward a target (the far cup) which is not reachable for her. Such behaviour is interpreted as an "ask" stimulus, eliciting a social complementary response of type "give" on the same target object, i.e. 
move the far cup closer to her, so that she can complete the action. No complex reasoning is required for this response, as common ask/give scenarios associating an action with a typical response are learnt by the associative memory so that, upon observation of an "ask" stimulus, a "give" response on the same target is automatic.

The filled square marker in Fig. 5 shows the switching point as detected by our model, while the filled round marker is the actual switching point observed in human studies. It can be observed how, even though the model is able to detect the change in the nature of the interaction before the end of the movement, the human subjects are much faster in this task. Almost identical results were obtained in a dual protocol in which precision grasping was substituting whole hand grasping 18 .

We believe there are three reasons for such discrepancy. The first is probably the limited accuracy of the model, probably in the correspondence between the proper and the observed kinematics parameter, which we are currently improving. The second limit is also something that can be dealt with, and is the quality of the sensory information provided to the model (e.g., very importantly, information on the wrist position, instead of the more revealing end effector). The third factor, which cannot really be overcome, is the natural social abilities of humans, which are able to take contextually into account a number of different aspects (posture, voice, gaze, sounds) which cannot be all included in the model.

\section{Employing the AOS/APS Model Framework in Human-Robot Interactions}

A final, long-term goal of this work is to endow an artificial system, as a humanoid robot, with more advanced social skills when engaged in interactions with human partners. In a previous work complementary to this, and also aimed at achieving the above described skills, we implemented a system for dynamic attention allocation able to actively control gaze movements during a visual action recognition task [17]. Similarly to what described for the reaching prediction in the cognitive science setup described above, the system is able to predict the goal position of the partner hand while it moves towards one of a number of visible targets. At the same time, robot gaze is controlled with the purpose of optimizing the gathering of information relevant for the task. An example of gazing behaviour by the robot, on a relatively cluttered virtual environment, can be observed in Fig. 6(a) Such skills have also been applied to actual interaction data, in which human movements are tracked and interpreted in real-time by the system, as shown in Fig. 6(b).

We are now extending the action prediction abilities shown by the system in the above described experiments for interpreting the possible social meaning of the partner's actions in order to prepare an appropriate response. This is done by introducing the social associative memory introduced in Sec. 4 to the system. Additionally, two contextual variables need to be taken into account: reachability and object identity. The first allows to discriminate what objects can 


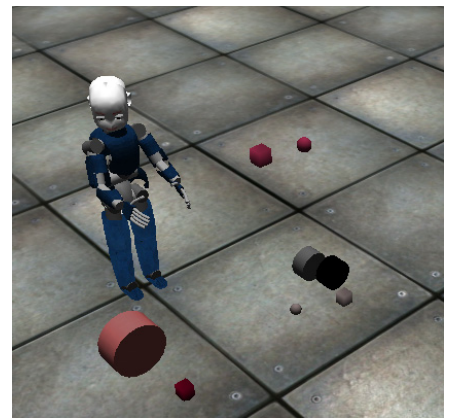

(a) Virtual experimental setup

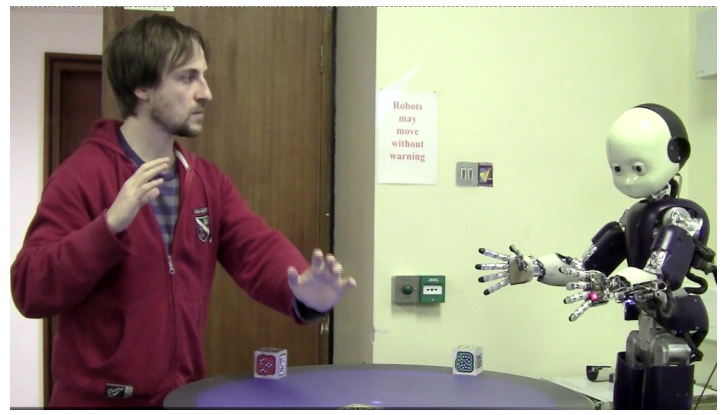

(b) Real world experimental setup

Fig. 6. Virtual and real experimental setups of robot gazing behaviour. In the virtual setup stimuli are represented by different coloured shapes. The red cylinder represents the robot gaze direction, see [17] for details. In the real setup stimuli are cube blocks.

be acted upon by either the subject and the partner, and the second introduces the environmental variability necessary to elicit different types of actions allowing to create a relatively complex instance of social memory.

Robotic implementation can represent a valuable testbed for the AOS/APS social interaction model, and at this stage we are able to advance some hypotheses of the effects we expect to observe by applying the model to real world interactions. First of all, the system good performance in action prediction (see [17]) should allow for a fast and reliable detection of the switching point between the AOS dominated resonance phase and the APS controlled social response. Second, we expect to observe a further improvement in such performance, consistently with the additional confidence the system can achieve in certain classes of social interactions, by practicing them. Finally, we plan to show with the robot a number of effects typically observed social interaction studies on human subjects, such as automatic imitation [13, cross-modal priming [12], interference [2] and familiarity effects [1].

\section{Conclusions}

A novel framework for modeling social interactions according to insights provided by cognitive science studies was presented in this work. It was shown how the framework is consistent with most findings, and how its implementation allowed us to replicate some effects observed in actual human studies. Methods and expected outcomes of applying the model to an existing robot system designed for endowing a humanoid robot with social skills were discussed. We are currently working on improving the performance of the model on various experimental neuroscience tasks, both on saved data and in real time. We are developing in parallel new skills for our robotic system thanks to the integration of different 
social frameworks. With all of the above, we aim at exploring further the nature of the relation between the AOS and APS systems, and the way they modulate their activity in order to generate final motor programs.

Acknowledgments. This research has received funding from the European Union Seventh Framework Programme FP7/2007-2013 - Challenge 2: Cognitive Systems, Interaction, Robotics - under grant agreement No [270490]- [EFAA], and from the University of Padova, under program Bando Giovani Studiosi 2011, L. n.240/2010.

\section{References}

1. Becchio, C., Manera, V., Sartori, L., Cavallo, A., Castiello, U.: Grasping intentions: from thought experiments to empirical evidence. Front Hum. Neurosci. 6, $117(2012)$

2. Blakemore, S.-J., Frith, C.: The role of motor contagion in the prediction of action. Neuropsychologia 43(2), 260-267 (2005)

3. Buccino, G., Binkofski, F., Fink, G.R., Fadiga, L., Fogassi, L., Gallese, V., Seitz, R.J., Zilles, K., Rizzolatti, G., Freund, H.J.: Action observation activates premotor and parietal areas in a somatotopic manner: an fmri study. Eur. J. Neurosci. 13(2), 400-404 (2001)

4. Cattaneo, L., Barchiesi, G., Tabarelli, D., Arfeller, C., Sato, M., Glenberg, A.M.: One's motor performance predictably modulates the understanding of others' actions through adaptation of premotor visuo-motor neurons. Soc. Cogn. Affect Neurosci. 6(3), 301-310 (2011)

5. Chinellato, E., Antonelli, M., Grzyb, B.J., del Pobil, A.P.: Implicit sensorimotor mapping of the peripersonal space by gazing and reaching. IEEE Transactions on Autonomous Mental Development (2011) (in press)

6. Chinellato, E., del Pobil, A.P.: Neural coding in the dorsal visual stream. In: Asada, M., Hallam, J.C.T., Meyer, J.-A., Tani, J. (eds.) SAB 2008. LNCS (LNAI), vol. 5040, pp. 230-239. Springer, Heidelberg (2008)

7. Chinellato, E., del Pobil, A.P.: The neuroscience of vision-based grasping: a functional review for computational modeling and bio-inspired robotics. Journal of Integrative Neuroscience 8(2), 223-254 (2009)

8. Costantini, M., Committeri, G., Sinigaglia, C.: Ready both to your and to my hands: mapping the action space of others. PLoS One 6(4), e17923 (2011)

9. Culham, J.C., Valyear, K.F.: Human parietal cortex in action 16(2), 205-212 (2006)

10. Demiris, Y., Khadhouri, B.: Hierarchical attentive multiple models for execution and recognition of actions. Robotics and Autonomous Systems 54, 361-369 (2006)

11. Fabbri-Destro, M., Rizzolatti, G.: Mirror neurons and mirror systems in monkeys and humans. Physiology (Bethesda) 23, 171-179 (2008)

12. Fadiga, L., Craighero, L., Olivier, E.: Human motor cortex excitability during the perception of others' action. 15(2), 213-218 (2005)

13. Heyes, C.: Automatic imitation. Psychol. Bull. 137(3), 463-483 (2011)

14. Jackson, P.L., Meltzoff, A.N., Decety, J.: Neural circuits involved in imitation and perspective-taking. Neuroimage 31(1), 429-439 (2006)

15. Jastorff, J., Begliomini, C., Fabbri-Destro, M., Rizzolatti, G., Orban, G.A.: Coding observed motor acts: different organizational principles in the parietal and premotor cortex of humans. J. Neurophysiol. 104(1), 128-140 (2010) 
16. Mukamel, R., Ekstrom, A.D., Kaplan, J., Iacoboni, M., Fried, I.: Single-neuron responses in humans during execution and observation of actions. Curr. Biol. 20(8), 750-756 (2010)

17. Ognibene, D., Chinellato, E., Sarabia, M., Demiris, Y.: Towards contextual action recognition and target localization with active allocation of attention. In: Prescott, T.J., Lepora, N.F., Mura, A., Verschure, P.F.M.J. (eds.) Living Machines 2012. LNCS, vol. 7375, pp. 192-203. Springer, Heidelberg (2012)

18. Sartori, L., Bucchioni, G., Castiello, U.: When emulation becomes reciprocity. Soc. Cogn. Affect Neurosci. (May 2012)

19. Sartori, L., Cavallo, A., Bucchioni, G., Castiello, U.: From simulation to reciprocity: the case of complementary actions. Soc. Neurosci. 7(2), 146-158 (2012)

20. Spunt, R.P., Lieberman, M.D.: The busy social brain: evidence for automaticity and control in the neural systems supporting social cognition and action understanding. Psychol. Sci. 24(1), 80-86 (2013)

21. Wilson, M., Knoblich, G.: The case for motor involvement in perceiving conspecifics. Psychol. Bull. 131(3), 460-473 (2005)

22. Wolpert, D.M., Doya, K., Kawato, M.: A unifying computational framework for motor control and social interaction. Philos. Trans. R. Soc. Lond. B. Biol. Sci. 358(1431), 593-602 (2003) 